\title{
TransSQL: A Translation and Validation-based Solution for SQL-Injection Attacks
}

\author{
Kai-Xiang Zhang, Chia-Jun Lin \\ Computer Science \& Info. \\ Engineering, \\ National Central University \\ Chungli, Taoyuan, Taiwan \\ $\{$ kxzang,cjlin $\} @$ adl.csie.ncu.edu.tw
}

\author{
Shih-Jen Chen \\ Network \& Multimedia Inst, Inst. \\ for Info. Industry \\ Taipei, Taiwan, R.O.C. \\ sjchen@iii.org.tw
}

\author{
Yanling Hwang \\ School of Applied Foreign \\ Languages \\ Chung Shan Medical University \\ Taichung, Taiwan, R.O.C. \\ yanling_h@yahoo.com
}

\author{
Hao-Lun Huang, Fu-Hau Hsu \\ Computer Science \& Info. \\ Engineering \\ National Central University \\ \{hlhuang,hsufh\}@adl.csie.ncu.edu.tw
}

\begin{abstract}
SQL injection attacks, a class of injection flaw in which specially crafted input strings leads to illegal queries to databases, are one of the topmost threats to web applications. A number of research prototypes and commercial products that maintain the queries structure in web applications have been developed. But these techniques either fail to address the full scope of the problem or have limitations. Based on our observation that the injected string in a SQL injection attack is interpreted differently on different databases, in this paper, we propose a novel and effective solution TransSQL to solve this problem. TransSQL automatically translates a $S Q L$ request to a LDAP-equivalent request. After queries are executed on a SQL database and a LDAP one, TransSQL checks the difference in responses between a SQL database and a LDAP one to detect and block SQL injection attacks. Experimental results show that TransSQL is an effective and efficient solution against $S Q L$ injection attacks.
\end{abstract}

Keywords: SQL injection; $L D A P$; web security

\section{INTRODUCTION}

SQL injection attacks refer to a class of attacks in which an adversary inserts specially crafted control code into the vulnerable field of a SQL query. SQL injection attacks allow attackers to gain control of the original query, unauthorized access to the database, and extract or modify sensitive information. In the last decade, web-based applications, such as on-line stores and social networks, have become a popular means to provide services to Internet users. Many of these applications utilize a database to provide their service. Hence SQL injection attacks soon become a popular approach for attackers to compromise a database. However, research [1, 2, 17] shows that the widespread deployment of firewalls and NIDSes can only provide limited protection to the majority of databases used by web-based applications.

In this paper we proposed an approach, detecting malicious SQL queries by distinct environment, a technique that automatically translates SQL queries into LDAPequivalent queries and validates the results from SQL databases and LDAP databases. In addition to core concept, we designed few extra-functions to deal with some special cases in our approach. Based on above ideas, we implement a tool called TransSQL (Translating SQL query to against SQL injection attacks). TransSQL offers several advantages. First, it is a server-side solution that does not need to modify/update the legacy web application. Second, the deployment of our technique is completely automatic, in other words, users can install TransSQL by "a click." Furthermore, we don't need to extract any information from applications source code in our technique. Third, although LDAP acts as an important role in TransSQL, users do not need to know any LDAP background knowledge such as LDAP schema and LDAP Data Interchange Format file. TransSQL has the ability to take over these stuffs. Finally, TransSQL is independent of the existing web application and database that provide the strong portability..

We support this claim by evaluating the large sets of SQL injection attacks and heavy testing of legitimate SQL queries. Our experimental results demonstrate effectiveness in preventing all classes of SQL injection attacks, and the protected web application could provide its service with no abnormal matter.

This paper makes the following contributions:

a). Proposed a novel solution to prevent SQL injection attacks that consists of database duplicating and runtime monitoring.

b). A fully automated approach with remarkable convenience in deployment.

c). A comprehensive evaluation of the effectiveness of attacks prevention.

The rest of the paper is organized as follows. We discuss our technique in Section 2. In Section 3 we review and discuss related work. Finally, we conclude the paper and outline future works in Section 4.

\section{DESIGN OF TRANSSQL}

In this section, we propose a novel technique, TransSQL, to solve the notorious SQL injection problem. TransSQL automatically duplicates a SQL database and stores the duplicated database in LDAP form. Then TransSQL monitors connections between the protected Web Application and the related SQL Database. Whenever a SQL request is sent to the SQL database, TransSQL creates a LDAP-equivalent request of the SQL request and sends the LDAP request to the LDAP database. TransSQL determines whether the SQL request is a SQL injection request or not by comparing the results from both the SQL database and the LDAP database.

\section{A. Overview of Trans $S Q L$}

Many of techniques ranging from source code development to web server framework have been proposed to detect and prevent SQL injection attacks. All these techniques either attempt to keep the integrity of SQL structure or examine the correctness of the SQL statements model. However, most proposed detection and prevention 
solutions have deployment problems. TransSQL counters SQL injection attacks through SQL request translation and SQL database duplication.

What follows are the major properties of TransSQL: 1). No need to modify or update the legacy application code. 2). No need to address the vulnerabilities in web applications. 3). No need to learn any background knowledge in SQL injection attacks and LDAP deployment.

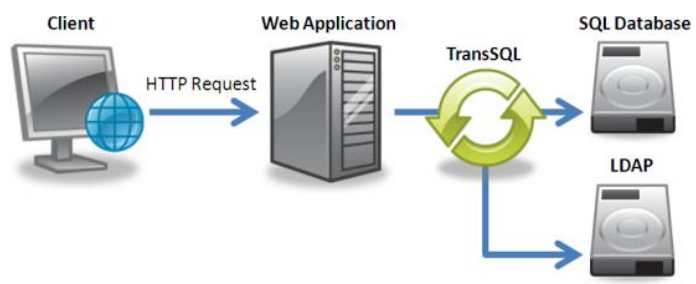

Figure 1: The framework of TransSQL.

TransSQL consists of two phases, preprocessing phase and runtime phase. In preprocessing phase, TransSQL retrieves information from a SQL database to produce corresponding LDAP schema and LDIF (LDAP Data Interchange Format) file. Then TransSQL builds a LDAPequivalent database by importing LDAP schema and LDIF data into the LDAP database. In runtime phase, TransSQL intercepts every SQL re-quest between the protected SQL database and a web application and translates it into LDAPequivalent request. A SQL request is a SQL injection request if the results re-turning from both databases have inconsistent responses. After detecting a SQL injection request, the result from SQL database would be replaced with a null result and send it to the web application. Figure 1 shows that the framework of protected web server. What follows summarizes TransSQL features. Detailed descriptions of them are given in subsequent sections.

- LDAP Features

Indicate LDAP inherent features to resist SQL injection attacks and how it operates in TransSQL.

- Database Duplication

Establish a general LDAP which contains identical information as existing SQL database by extracting table data from sqldump file and manufacturing the formal LDAP schema and LDIF files.

- SQL Request Translation

Each SQL request is translated into a LDAP-equivalent request.

- Special Cases

In addition to fundamental defense mechanism, TransSQL is directed against certain types of SQL injection attacks and emerging leak.

\section{B. Database Duplication}

Our proposed technique prevents SQL injection attacks by querying both SQL database and LDAP. Therefore, ensuring the equivalent contents of SQL database and LDAP becomes an important work. As Figure 2, SQL database is composed by rows, tables, or other database elements, but LDAP is composed by entries and attributes. It seems completely different model in theory, but both of them have many common functions such as query and storage.

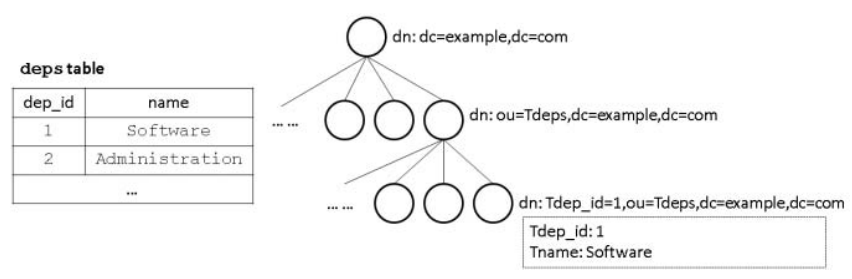

Figure 2: Database Duplication Example. On the left is the SQL table example, and on the right is the equivalent data in LDAP.

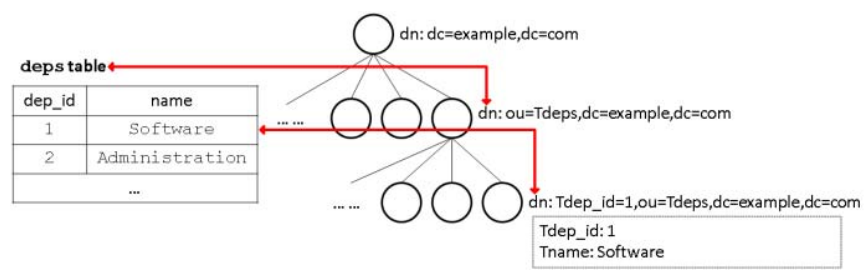

Figure 3: A data in SQL database corresponding to an entry in LDAP.

In order to accomplish schema definition we defined corresponding rules as the following.

i). Append signature to table name and column name during duplicating. Collisions may happen to duplicating process if TransSQL just duplicates constant table name and column name to LDAP schema. The reason is that name of objectClasses and attributes probably had been defined in existing schema so that the present objectClasses, attributes name couldn't be defined again. Therefore, objectClasses and attributes name is mixed with signature which is given by users and table name, column name. (e.g., the signature is $\mathrm{T}$, so that the original column name name becomes Tname.)

ii). Tables in SQL database become objectClasses in LDAP. LDAP objectclasses which are defined inside schemas are the means for including attributes (they are an attribute containers in the jargon). TransSQL treats a table in SQL database as an objectClass in LDAP, and examines what columns the table has. LDAP objectclasses define whether an attribute is mandatory (MUST be present) or optional (MAY be present) as good as table columns can be defined for NOT NULL or default NULL.

iii). Columns in SQL database become attributes in LDAP. As Column definition in SQL database, an attribute definition includes its type (or SYNTAX), for example, a string or number. There are still many operations on attributes, but TransSQL only determines EQUALITY which is necessary in duplicating process.

iv). Each of rows is assigned a distinguished name and becomes an entry under the table entries. Each entry has a unique identifier, distinguished name (DN). This consists of its relative distinguished name (RDN), constructed from some attribute(s) in the entry, followed by the parent entry's DN. Think of the DN as the full file path and the RDN as its relative filename in its parent folder (e.g. if 
$\mathrm{ou}=$ users, $\mathrm{dc}=$ example, $\mathrm{dc}=\mathrm{com}$ were the $\mathrm{DN}$, then $\mathrm{ou}=$ users would be the RDN). TransSQL manufactures unique identifier as RDN for each row. Every table rows convert into child entries of table entry in LDAP as Figure 3.

v). A additional file records columns in a table.

\section{SQL Request Translation}

In order to prevent SQL injection attacks and preserve functioning of web application, the translation process is a significant part for TransSQL. To translate SQL requests into LDAP-equivalent requests is a heavy issue due to SQL complicated syntax.

We use Zql library to parse SQL. Zql parses SQL and fills in java structures representing SQL statements and expressions. Besides SQL parser, there is another efficient technique to transform SQL requests into LDAP-equivalent requests. To do this, we use the JDBC-LDAP library [16]. This technique (JDBC->LDAP bridge) provides a JDBC interface to use SQL when interacting with database services and utilizes JLDAP to interact with LDAP. This bridge has adapted the common SQL commands such as SELECT, INSERT, DELETE and UPDATE along with a JDBCLDAP Bridge specific command called UPDATE ENTRY. LDAP request is similar to SQL request by using JDBCLDAP technique. For instance, the following is a SQL query.

SELECT * FROM users WHERE name=' john'

The LDAP-equivalent query presented as below.

SELECT * FROM ou=users, dc=example, dc=com

WHERE name=' john'

The major difference between standard SQL and the above commands is the use of LDAP DNs. In general, TransSQL only needs to replace table by DN, but there is a variety of SQL expressions in web applications. The translation process should be as completely as possible due to keep present web applications operating ordinarily. Except for replacing table by $\mathrm{DN}$, we enhanced translation process to deal with SQL which is unable to translate directly, for instance, alias, UNION and UNION ALL, etc. The part of SQL commands, UNION, UNION ALL and so on, are not supported by JDBC-LDAP library, so that TransSQL splits them into two requests and combines both results. The criterion of translation process is stand on passing Zql's heavy testing which is a benchmark used for SQL parser's quality assurance.

\section{RELATED WORK}

Researchers have been intense research in detection and prevention mechanisms against SQL injection attacks recently. These techniques range from source code development to web server framework so that we can classify these approaches broadly into three subjects in this section.

\section{A. Coding Practices}

Many defensive mechanisms can be used by programmers during the development phase. These coding practices include extensive input validation and the usage of PREPARE statements. The input validation is a function in general. For instance, there is a function, mysql real escape string() [3], provided by mysql library in PHP. It can escape special characters in a string for use in an SQL statement. This mechanism is an arduous task because the programmer must make sure that all strings have been escaped before sending a query to SQL database. PREPARE statements which separate the values in a query from the structure of SQL. Using PREPARE statements is very effective against SQL injection attacks. Hibernate [4] enforces developers to use the PREPARE statement. The problem is that rewrites legacy web applications to PREPARE statements is hard to automate and not viable. Two similar approaches, McClure et al. proposed SQL DOM [5] and Cook et al. proposed Safe Query Objects [20], use encapsulation of database queries to provide a safe way to access database. These techniques we discuss above have the disadvantage that developers have to learn and use a new programming paradigm.

\section{B. Application Analysis}

Huang et al. proposed WAVES [6], a black-box technique used to test web applications for SQL injection vulnerabilities. WAVES is a web crawler that identifies all points in a web application that can be used to inject malicious code. The technique raises attacks that target those points based on a list of patterns and attack techniques. WAVES is better than traditional penetration testing, because it improves the attack methodology by machine learning technique. However, it cannot thoroughly check all the vulnerable points and provide guarantees of completeness. Kosuga et al. proposed Sania [7], the technique intercepts SQL queries and automatically generates elaborate attacks based on the syntax of potentially vulnerable points in the SQL queries. Sania assesses the safety by comparing the parse trees of the original SQL query and that resulting after the attack. Unfortunately, certain types of obfuscation codes or query generation technique make Sania less precise and results in both false positives and negatives.

Livshits et al. and Xie et al. [8,9] perform static analysis over the entire application's source code to ensure that every piece of input is subject to an input validation check. These techniques are limited to identify sources (points of input) and sinks (query issuing locations). Typical precision issues with static analysis, especially when checking dynamically constructed queries, indicate that they may identify several illegal flows in a web application, even if these paths are infeasible. In addition, they do not help users determine whether the sanitization routines prevent SQL injection attacks.

\section{Defense framework}

These techniques that prevent SQL injection attacks are significantly different from vulnerability analysis as we 
discuss previously. They do not demand the programmer to perform input validation to prevent injection attacks, and hence offer effective solutions for securing applications including legacy code.

Halfond et al. proposed AMNESIA that is a model-based technique combines static analysis and runtime monitoring $[10,11]$. In the static phase, AMNESIA uses a static analysis to build the models of the SQL queries that an application legally generates at each point of access to the database. In the dynamic phase, AMNESIA intercepts all queries before they are sent to the database and checks each query against the statically built models. Queries that violate the model are identified as SQL injection attacks. The accuracy of AMNESIA depends on the static analysis. Unfortunately, web application programs may use conditional branching heavily to dynamically construct SQL queries, and that could make this technique less precise and result in both false positive and false negatives.

Two recent similarly approaches, Buehrer et al. proposed SQLGuard [12] and Su et al. proposed SQLCheck [13], also check queries at runtime to see if they conform to a model of expected queries. In these approaches, the model is expressed as a grammar that only accepts legal queries. In SQLGuard, the model is deduced at run-time. In SQLCheck, the model is specified independently by the developer. The use of these two approaches requires the developer to either rewrite code to use a special intermediate library or manually insert special markers into code where user input is added to a dynamically generated query.

Pietraszek et al. proposed an approach [14], they modified a PHP interpreter to track taint information at the character level. This technique use a context-sensitive analysis to reject SQL queries if an malicious input has been used ti create certain types of SQL tokens. The most important disadvantage of this approach is that they require modifications to the runtime environment, which diminished the portability. Boyd et al. proposed SQLrand [15] that allows developers to create SQL queries using randomized keywords instead of the normal SQL keywords. The SQL keywords injected by an attacker would not have been constructed by the randomized keywords, and thus the injected commands would result in a syntactically incorrect query.

\section{CONCLUSION}

We have presented a novel fully automated technique, TransSQL, for preventing SQL injection attacks. The technique is based on the intuition that injection codes implicitly perform a different meaning from general queries. We presented an elaborate environment based on LDAP for distinguishing legitimate and malicious queries. To complete this task, TransSQL is consisted with preprocessing step and runtime step. In the preprocessing step, the technique uses an existing SQL command to extract from SQL database a file which contains whole information of SQL database. According to the sqldump file, TransSQL generates a duplicated database in LDAP form. In runtime step, TransSQL monitors connection between web applications and SQL databases. Every query would be translated into a LDAP-equivalent query, and then we defined some conditions to identify malicious queries.

\section{REFERENCES}

[1] C. Anley. Advanced SQL Injection In SQL Server Applications. White paper, Next Generation Security Software Ltd., 2002.

[2] C.Anley. (more) Advanced SQL Injection. White paper, Next Generation Security Software Ltd., 2002.

[3] MySQL Library. mysql_real_escape_string() function. http://php.net/manual/en/function.mysql-real-escape-string.php

[4] Hibernate. hibernate.org. http://www.hibernate.org/.

[5] R. McClure and I. Kr uger. SQL DOM: Compile Time Checking of Dynamic SQL Statements. In Proceedings of the $27^{\text {th }}$ International Conference on Software Engineering (ICSE 05), 2005.

[6] Y. Huang, S. Huang, T. Lin, and C. Tsai. Web Application Security Assessment by Fault Injection and Behavior Monitoring. In Proceedings of the $11^{\text {th }}$ International World Wide Web Conference (WWW 03), 2003.

[7] Y. Kosuga, K. Kono, M. Hanaoka, M. Hishiyama, and Y. Takahama. Sania: Syntactic and Semantic Analysis for Automated Testing against SQL Injection. In Proceedings of the $23^{\text {rd }}$ Annual Computer Security Applications Conference (ACSAC 07), 2007.

[8] V. B. Livshits and M. S. Lam. Finding Security Errors in Java Programs with Static Analysis. In Proceedings of the $14^{\text {th }}$ Usenix Security Symposium, 2005.

[9] Y. Xie, and A. Aiken. Static detection of security vulnerabilities in scripting languages. In Proceedings of the $15^{\text {th }}$ Conference on USENIX Security Symposium. 2006.

[10] W. G. Halfond and A. Orso. AMNESIA: Analysis and Monitoring for NEutralizing SQL-Injection Attacks. In Proceedings of the IEEE and ACM International Conference on Automated Software Engineering (ASE 2005), 2005.

[11] W. G. Halfond and A. Orso. Combining Static Analysis and Runtime Monitoring to Counter SQL-Injection Attacks. In Proceedings of the Third International ICSE Workshop on Dynamic Analysis (WODA 2005), 2005.

[12] G. T. Buehrer, B. W. Weide, and P. A. G. Sivilotti. Using Parse Tree Validation to Prevent SQL Injection Attacks. In International Workshop on Software Engineering and Middleware (SEM), 2005.

[13] Z. Su and G. Wassermann. The Essence of Command Injection Attacks in Web Applications. In The $33^{\text {rd }}$ Annual Symposium on Principles of Programming Languages (POPL 2006), 2006.

[14] T. Pietraszek and C. V. Berghe. Defending Against Injection Attacks through Context-Sensitive String Evaluation. In Proceedings of Recent Advances in Intrusion Detection (RAID2005), 2005.

[15] S. W. Boyd and A. D. Keromytis. SQLrand: Preventing SQL Injection Attacks. In Proceedings of the 2nd Applied Cryptography and Network Security (ACNS) Conference, 2004.

[16] MySQL. mysqldump - A Database Backup Program. http://dev.mysql.com/doc/refman/5.1/en/mysqldump.html.

[17] My Virtual Directory. JDBC->LDAP Bridge. http://myvd.sourceforge.net/jdbcldap.html, 2008

[18] S. McDonald. SQL Injection: Modes of attack, defense, and why it matters. White paper, GovernmentSecurity.org, 2002. 